\title{
Avanafil for treatment of erectile dysfunction: review of its potential
}

\author{
This article was published in the following Dove Press journal: \\ Vascular Health and Risk Management \\ 28 August 2012 \\ Number of times this article has been viewed
}

\author{
Ryan M Burke' \\ Jeffery D Evans² \\ 'The University of Louisiana at \\ Monroe College of Pharmacy, \\ Monroe, LA, ${ }^{2}$ The University of \\ Louisiana at Monroe College of \\ Pharmacy, Shreveport, LA, USA
}

Correspondence: Jeffery D Evans University of Louisiana at Monroe, College of Pharmacy, Shreveport Campus, 1725 Claiborne Ave, Shreveport, LA 7II03, USA

Email jevans@ulm.edu
Abstract: Avanafil is a medication that was recently approved by the US Food and Drug Administration for the management of erectile dysfunction. Avanafil is a new phosphodiesterase type 5 inhibitor similar to sildenafil and tadalafil. Avanafil was studied in over 1300 patients during clinical trials, including patients with diabetes mellitus and those who had undergone radical prostatectomy, and was found to be more effective than placebo in all men who were randomized to the drug. The medication was studied with on-demand dosing that may occur after food and/or alcohol. Avanafil is dosed as $50 \mathrm{mg}, 100 \mathrm{mg}$, or $200 \mathrm{mg}$ tablets. Avanafil may differentiate itself from the other phosphodiesterase type 5 inhibitors with its quicker onset and higher specificity for phosphodiesterase type 5 versus other phosphodiesterase subtypes, but may lead to complications of therapy.

Keywords: avanafil, safety, efficacy, erectile dysfunction

\section{Introduction}

Avanafil was approved by the US Food and Drug Administration for the treatment of erectile dysfunction on April 27, 2012. ${ }^{1}$ The medication will be marketed in the US as Stendra ${ }^{\circledR}$ (Vivus, Mountain View, CA). The Food and Drug Administration has approved avanafil in dose strengths of $50 \mathrm{mg}, 100 \mathrm{mg}$, and $200 \mathrm{mg}$. Patients should take the medication as needed to achieve an erection and should be prescribed the lowest effective dose. The medication was approved on the basis of three trials that showed a benefit over placebo. An application to market avanafil has been completed and was submitted to the European Union in March 2012. The medication is also currently approved for use in South Korea under a different brand name.

Determining rates of erectile dysfunction is difficult due to a potentially low level of reporting by survey responders and a lack of clear definition of erectile dysfunction provided to the men completing the surveys. The incidence of erectile dysfunction increases with increasing age. ${ }^{2}$ Men aged 18-59 years have an incidence of erectile dysfunction of $10 \%$. Other data looking at similar groups divided the age groups into 10-year sections and found that, by setting individuals aged 18-29 years as a reference group, individuals aged 50-59 years had a 3.5 times higher incidence of erectile dysfunction. Another survey found that males older than 70 years had a $61 \%$ incidence of erectile dysfunction

Erectile dysfunction may be classified as organic, psychogenic, or a mixture of both. Organic includes erectile dysfunction caused by neurologic factors, penile structure, 
vascular abnormalities, or medications. ${ }^{3}$ Psychogenic erectile dysfunction may be associated with several factors, but is frequently linked to performance anxiety, lack of libido, or other psychiatric conditions, such as depression. In purely psychogenic erectile dysfunction, the patient will report that they are able to obtain an erection at times not related to sexual activity but not during periods of sexual stimulation. Neurologic diseases, such as Parkinson's disease or Alzheimer's disease, may also lead to erectile dysfunction by decreasing the patient's libido or by inhibiting the signaling to start an erection. Advancing age may lead to both a decrease in testosterone levels and a change in the vasculature of the penis. Finally, the risk of erectile dysfunction increases with increasing number of medications being taken, especially blood pressure-lowering medication. Other medications, such as selective serotonin reuptake inhibitors, decrease libido in some patients, which may lead to erectile dysfunction.

Erectile dysfunction may also play a role as an indicator of other diseases. Batty et al studied men with and without erectile dysfunction and diabetes mellitus, and found that men with both erectile dysfunction and diabetes mellitus were more likely to have coronary heart disease or cerebrovascular disease. ${ }^{4}$ This trial was a subset analysis of ADVANCE (Action in Diabetes and Vascular Disease: Preterax and Diamicron Modified Release Controlled Evaluation) and was cohort-controlled. Dong et $\mathrm{al}^{5}$ completed a meta-analysis that included about 36,000 patients, and after controlling for other demographics, found that men with erectile dysfunction were more likely to have heart disease and had a statistically higher incidence of all-cause mortality.

A diagnosis of erectile dysfunction is made when a man has a consistent or recurrent inability to either obtain or maintain an erection allowing for completion of sexual activity. ${ }^{6}$ Usually the diagnosis of erectile dysfunction should be made after at least 3 months of symptoms; however, if some type of trauma occurred prior to the patient developing symptoms, the patient may be diagnosed with erectile dysfunction in a shorter time frame. Patients can be diagnosed according to self-reported symptoms or by physiologic testing. If necessary, partner reporting may be used to initiate the process of screening a patient for erectile dysfunction.

The initial diagnosis of erectile dysfunction may be delayed by the patient's reluctance to answer questions or to initiate a conversation with their health care provider. It is important for the patient to feel comfortable discussing their symptoms during a consultation. Other important information that must be collected includes a medical history and a medication history. Several diseases, including cardiovascular disease, may cause or worsen erectile dysfunction. Unfortunately, many medications, including those used to treat cardiovascular disease, may also cause or worsen the problem. In addition to physiologic causes, psychologic causes such as anxiety or relationship issues, may lead to erectile dysfunction.

Objective data are also collected. Patients presenting with erectile dysfunction will need to be screened for other diseases, including diabetes mellitus and cardiovascular disease. Also, specific laboratory tests for testosterone and certain endocrine disorders, such as thyroid dysfunction, should be completed and evaluated to determine if there are secondary causes for erectile dysfunction.

\section{Review of current therapies}

Current American Urology Association guidelines include oral phosphodiesterase type 5 inhibitors (sildenafil, tadalafil, vardenafil), localized alprostadil (intraurethral suppositories, intracavernous injection), testosterone, and vacuum constriction devices as viable nonsurgical options for the treatment of erectile dysfunction.

\section{Phosphodiesterase type 5 inhibitors}

American Urology Association guidelines recommend phosphodiesterase type 5 inhibitors as first-line therapy for erectile dysfunction. ${ }^{7}$ These agents are orally active and self-administered on an as-needed basis prior to sexual intercourse. Major clinical differences between these compounds are related to their different pharmacokinetic profiles, primarily to time to onset and duration of action. Sildenafil citrate $\left(\right.$ Viagra $^{\circledR}$, Pfizer Inc, New York, NY) is rapidly absorbed after oral administration and reaches peak plasma concentrations within 30-60 minutes. Although absorption studies indicate that nearly $92 \%$ of the oral dose of sildenafil is absorbed, extensive gut wall and hepatic firstpass metabolism contribute to a relatively low bioavailability of $38 \%-41 \%$. High-fat meals contribute to a reduced rate of absorption by nearly one hour and a $29 \%$ decrease in systemic exposure of sildenafil. The plasma half-life of sildenafil is approximately 4 hours and the duration of action is nearly 12 hours. $^{8}$

Tadalafil (Cialis ${ }^{\circledR}$, Eli Lilly, Indianapolis, IA) is rapidly absorbed after oral administration its absorption and pharmacodynamic properties are not affected by food or alcohol. Clinical effects are typically observed 60-120 minutes after administration. ${ }^{9}$ Oral bioavailability is estimated to be at least $36 \%$ of the administered dose, and plasma half-life is 17.5 hours. 
Vardenafil (Levitra ${ }^{\circledR}$, Bayer, Leverkusen, Germany), unlike sildenafil and tadalafil, was developed with the intention of being used specifically as an erectogenic agent. It is rapidly absorbed following oral administration, with plasma peak plasma levels detected within one hour. The absolute bioavailability of vardenafil is approximately $15 \%$, which can be attributed to presystemic and hepatic first-pass metabolism. Although high-fat meals do not significantly affect the area under the concentration-time curve (AUC), mean peak plasma concentration is approximately $18 \%$ lower and median time to peak plasma concentration is delayed by one hour. The half-life of vardenafil is approximately 4 hours. ${ }^{10}$

\section{Localized therapy}

Alprostadil is another agent that can be used for multiple etiologies of erectile dysfunction. ${ }^{6}$ Alprostadil is available in two forms, ie, an intracavernosal injection or an intraurethral suppository. Alprostadil is a synthetic prostaglandin E1 analog that works in a manner similar to that of the phosphodiesterase type 5 inhibitors, in that it causes dilation of smooth muscle, trapping and allowing blood to collect in the penis, leading to erection. Due to its mode of application, it is not favored by most patients and is not generally recommended. However, patients who cannot tolerate any of the phosphodiesterase type 5 inhibitors may utilize these medications.

The other medication recommended for treatment of erectile dysfunction is testosterone. Testosterone has only been shown to be useful in patients who have a low testosterone level. Several formulations of testosterone are available, including gels and patches. Although testosterone used at appropriate doses generally has tolerable side effects, benign prostatic hyperplasia and prostate cancer are both concerns with use of this medication. Unlike other medications recommended for erectile dysfunction, testosterone must be used daily to have adequate efficacy for erectile dysfunction.

\section{Avanafil}

Avanafil (4-[(3-chloro-4-methoxybenzyl)amino]-2-[2(hydroxymethyl)-1-pyrrolidinyl]-N-(2-pyrimidinylmethyl)5-pyrimidinecarboxamide;(S)-2-(2-hydroxymethyl1-pyrrolidinyl)-4-(3-chloro-4-methoxybenzylamino)-5-[(2pyrimidinylmethyl) carbamoyl]pyrimidine) has a molecular weight of $483.95 \mathrm{Da}$ and is a pyrimidine derivative that exists as a single enantiomer with S stereochemistry. It appears as a white crystalline powder in its pure form and is minimally soluble in water and moderately soluble in organic solvent.
Avanafil is more soluble in acidic buffers (about $\mathrm{pH} 4$ ) and is much less soluble in neutral and alkaline buffers. ${ }^{9}$ Avanafil works in penile tissue to increase the level of cyclic guanosine monophosphate (cGMP). As levels of cGMP increase, smooth muscles relax in the penis, increasing local blood flow, which results in an erection. However, erection is only attained if stimulation occurs.

Avanafil has been designed to be a fast-acting and highly selective phosphodiesterase type 5 inhibitor. ${ }^{11}$ In a study comparing enzymatic inhibition of the phosphodiesterase isoenzymes for avanafil and sildenafil, both drugs showed phosphodiesterase type 5 isolated from canine lung in a dose-dependent manner. ${ }^{9}$ However, avanafil inhibited phosphodiesterase types 6 and 1 to a lesser extent than sildenafil. ${ }^{11}$ Based on the results of electroretinogram studies to determine the degree of phosphodiesterase type 6 inhibition, avanafil is unlikely to affect retinal function at pharmacologically appropriate doses.

Previous sildenafil studies have concluded that coadministration of sildenafil with organic nitrates can lead to significant and potentially dangerous hypotension. While both sildenafil and avanafil potentiate nitroglycerininduced hypotension, avanafil does so to a lesser degree than sildenafil. Similarly, both drugs potentiate sodium nitroprusside-induced inhibition of platelet aggregation, but to a lesser extent with avanafil than with sildenafil. ${ }^{9}$

Avanafil undergoes significant biotransformation via the human cytochrome P450 (CYP) enzyme system. ${ }^{11}$ More specifically, CYP3 A4 is primarily responsible for the metabolism of avanafil into at least 11 metabolites, with minor contributions from CYP2C. Phosphodiesterase type 5 inhibition of the metabolites is significantly less than that of the parent compound. ${ }^{9}$

Herbal medications are not generally recommended for first-line treatment of erectile dysfunction, but some patients may seek out these treatments. Yohimbine has been shown to be somewhat effective in the management of erectile dysfunction after 7-8 weeks of treatment. ${ }^{12}$ Yohimbine is an alpha-2-adrenergic receptor antagonist causing smooth muscle relaxation within the penis. At higher doses, there is also monoamine oxidase inhibition that can lead to significant side effects. Otherwise, the medication is tolerated by patients, with tachycardia, anxiety, and irritability being the common side effects. Red ginseng (Korean Panax) has also been shown to be effective for the treatment of erectile dysfunction when taken daily for up to 12 weeks. The most frequently reported side effects include insomnia and headache. Some caution should be exercised in patients 
with diabetes mellitus because it may cause hypoglycemia. L-arginine has been studied for organic erectile dysfunction and was originally thought to be effective. However, more recent, better controlled, and well powered studies have not found a significant decrease in symptoms of erectile dysfunction using L-arginine.

\section{Materials and methods}

In order to determine the potential advantages and disadvantages of avanafil, a literature search was performed using PubMed with the search terms "avanafil" and "erectile dysfunction". For the purposes of determining the safety, efficacy, and tolerability of avanafil, three randomized, double-blind, placebo-controlled clinical trials were selected for inclusion in this review. An additional search was performed using the terms "sildenafil", "tadalafil", and "vardenafil" to obtain safety, efficacy, and tolerability data for the three marketed phosphodiesterase 5 inhibitors, with the purpose of comparing these data with those for avanafil. Further, the information submitted with the New Drug Application to the US Food and Drug Administration was reviewed for possible unpublished trials.

\section{Results}

Three controlled, prospective trials were identified that contained data on the use of avanafil for the treatment of erectile dysfunction. Further, two trials were found to be included in the New Drug Application that were as yet unpublished.

\section{Study I}

The first was a Phase I double-blind, randomized, placebocontrolled, parallel-group, dose-escalation study performed by Jung et $\mathrm{al}^{13}$ to assess the tolerability and pharmacokinetics of avanafil. Healthy male volunteers, aged 18-24 years, weighing $>45 \mathrm{~kg}$, and within $\pm 20 \%$ of ideal body weight, were enrolled in the study and evaluated to determine their health status. Evaluation included assessment of medical history, physical examination, 12-lead electrocardiogram (ECG), visual color discrimination tests, and hematology, blood chemistry, and urinalysis. Exclusion criteria were evidence of any clinically significant disease or abnormality, prescription medication use in the 2 weeks preceding the study, a history of diabetic retinopathy or retinitis pigmentosa, and presence of acute illness, or hematologic, cardiovascular, or psychologic disorders.

Patients were randomly assigned to one of three avanafil dose groups (50, 100, or $200 \mathrm{mg}$ ). Each group consisted of ten patients; eight received avanafil and two received placebo. Doses were administered at 24-hour intervals for 7 days. Blood samples were collected once before and multiple times after drug administration on days 1 and 7 to assess the pharmacokinetics of avanafil. The mean time to peak plasma concentration for avanafil was $0.33-0.52$ hours and the mean elimination half-life was 5.36-10.66 hours. There were no significant differences between the pharmacokinetic results obtained on days 1 and 7. Dose proportionality was observed, and there was no significant difference between the predicted and observed AUC values for subjects receiving avanafil 50, 100 , or $200 \mathrm{mg}$.

Analysis of hematology, blood chemistry, and urinalysis laboratory results, vital signs, 12-lead ECG, and color discrimination test results were utilized to assess the tolerability of avanafil. Investigators were monitored for adverse events by spontaneous reporting and specific questioning throughout the study period. Adverse events were reported by 20 of 25 patients in the avanafil treatment groups and by four of six patients in the placebo group. The treatment groups and the placebo group reported flushing, headache, and dizziness. Additional adverse events reported by the avanafil groups included orbital pain, chest discomfort, epigastric heartburn, nasal congestion, abdominal discomfort, chest discomfort, and paresthesia. All adverse events were considered to be mild, and resolved spontaneously. Investigators observed no clinically relevant changes in vital signs, ECG recordings, physical examination findings, or color discrimination test results.

The researchers concluded that avanafil was generally well tolerated and had linear pharmacokinetic properties at daily doses of 50-200 mg over 7 days. They also pointed out that further studies are warranted, given that this study involved only Korean subjects and had a relatively small sample size of 30 subjects.

\section{Study 2}

There are few trials that have studied the safety and efficacy of avanafil for the treatment of erectile dysfunction in male subjects. This Phase III, prospective, multicenter, randomized, double-blind, placebo-controlled, parallelgroup evaluation of avanafil for the treatment of erectile dysfunction was performed by Goldstein et al. ${ }^{14}$ In order to be included in this trial, men had to be aged 18 years or older and have a 6-month or longer history of mild to severe erectile dysfunction. Patients were excluded if they had known allergy or hypersensitivity to avanafil, sildenafil, vardenafil, tadalafil, or any of their components, a history of 
dose-limiting adverse events during previous treatment with a phosphodiesterase type 5 inhibitor, or a history of use of any agent known to inhibit CYP3A4 within 28 days prior to randomization. Other exclusion criteria included current or expected use of organic nitrates during the study, androgen replacement therapy that had not been stable for $\geq 3$ months, erectile dysfunction as a result of spinal cord injury or radical prostatectomy, untreated hypogonadism, a history of or predisposition to priapism, a penile implant, prostatespecific antigen level $>4 \mathrm{ng} / \mathrm{mL}$ or other evidence of prostate cancer, diabetes, uncontrolled hypertension, hypotension, myocardial infarction, stroke, life-threatening arrhythmia, or coronary revascularization within the past 6 months. Patients were eligible for randomization to a treatment arm if they had a $\geq 50 \%$ failure rate in maintaining erections of sufficient duration to allow for successful intercourse; had an International Index of Erectile Function (IIEF) erectile function (EF) domain score of 5-25, inclusive; and had made at least four attempts at sexual intercourse during the initial 4-week, nontreatment, run-in period. A total of 646 patients were randomized to receive $50 \mathrm{mg}, 100 \mathrm{mg}$, or $200 \mathrm{mg}$ of avanafil, or placebo, for a 12 -week treatment period.

The study had three coprimary efficacy endpoints: change in percentage of sexual attempts in which subjects were able to insert the penis into the partner's vagina between the run-in period and the end of the 12-week treatment period; change in percentage of attempts in which subjects were able to maintain an erection of sufficient duration to have successful intercourse between the run-in period and the end of the 12-week treatment period; and change from baseline to end of treatment in IIEF-EF domain score.

With regard to coprimary endpoint 1, treatment with avanafil enabled significant improvement compared with placebo, the $50 \mathrm{mg}$ dose was superior to both the $100 \mathrm{mg}$ and $200 \mathrm{mg}$ doses, while no significant difference was observed between the $100 \mathrm{mg}$ and $200 \mathrm{mg}$ doses. With regard to coprimary endpoint 2 , the placebo group experienced a $27 \%$ mean change from baseline; the $50 \mathrm{mg}$ avanafil group experienced a $41 \%$ change; and the $100 \mathrm{mg}$ and $200 \mathrm{mg}$ avanafil groups experienced at 57\% change. Similarly, for coprimary endpoint 3 , the three treatment groups showed significant improvement in the IIEF-EF domain score compared with placebo. Commonly reported adverse effects included headache, flushing, nasal congestion, back pain, nasopharyngitis, and bronchitis.

The researchers concluded that avanafil is well tolerated and effective for the treatment of mild to severe erectile dysfunction. They also concluded that treatment responses were reported as early as 15 minutes following a dose, with some patients reporting effects more than 6 hours after a dose.

\section{Study 3}

This Phase III, multicenter, randomized, double-blind, placebo-controlled trial by Zhao et $\mathrm{al}^{15}$ evaluated the safety and efficacy of avanafil for the treatment of erectile dysfunction in 200 male subjects. The study was conducted at 14 different centers in Korea. Individuals included were men with erectile dysfunction of at least 6 months in duration, $>20$ years of age, in a stable heterosexual relationship for at least 6 months, and who had made at least four attempts at sexual intercourse during the 4-week run-in period, of which at least $50 \%$ of attempts were unsuccessful. Patients excluded were those with anatomical penile defects, spinal cord injury, previous radical prostatectomy or radical pelvic surgery, hyperprolactinemia, low total testosterone, poorly controlled diabetes $\left(\mathrm{HbA}_{1 \mathrm{c}}>12 \%\right)$ or proliferative diabetic retinopathy, major uncontrolled psychiatric disorder, history of active peptic ulcer disease, history of major hematologic, renal or hepatic abnormalities, history of cardiovascular disease, stroke, myocardial infarction, cardiac failure, unstable angina, or life-threatening arrhythmia in the past 6 months, or a history of alcoholism or substance abuse. Additional exclusion criteria were: regular use of nitrates, anticoagulants (except for aspirin), androgens, antiandrogens, or trazodone; use of erythromycin, cimetidine, ketoconazole, indinavir, or grapefruit juice; and failure of previous phosphodiesterase type 5 inhibitor therapy.

Patients were randomly assigned to receive placebo, avanafil $100 \mathrm{mg}$, or avanafil $200 \mathrm{mg}$, and were allowed to take the product with water when necessary 30 minutes before sexual intercourse. The study used the change in baseline in erectile dysfunction domain (EFD) scores of the IIEF questionnaire as the primary efficacy endpoint. Secondary efficacy endpoints included patient responses to questions from the Sexual Encounter Profile (SEP), which assessed ability to insert the penis into the partner's vagina and maintain an erection of sufficient duration to complete intercourse. These endpoints were assessed after each attempt at intercourse with avanafil or placebo. Researchers also assessed patient responses to the global assessment question (GAQ, "Has the treatment you have been taking during the study improved your erections?") after 12 weeks of treatment.

In total, 200 patients were included in the intent-to-treat analysis. Analyses of differences between visits revealed 
that both avanafil groups displayed statistically significant improvement with regard to change from baseline in EFD scores on the IIEF, while the placebo group showed no significant improvement. Similarly, when the baseline value was compared between visits, both avanafil groups showed statistically significant improvements, and the placebo group did not. No significant differences were observed between the $100 \mathrm{mg}$ and $200 \mathrm{mg}$ avanafil groups. There were significant changes from baseline in each domain of the IIEF in the avanafil groups compared with placebo. Further, comparison of rates of response to SEP, the proportion of "yes" responses to the GAQ, and the percentage of patients achieving normal EFD scores, showed that all patients in the avanafil groups experienced a significant difference, while the placebo group did not.

This trial concluded that avanafil was generally safe and well tolerated with only mild to moderate adverse effects. There was one report of visual abnormality, but the rate was considered much lower than that with other currently available phosphodiesterase type 5 inhibitors. The investigators concluded that the higher selectivity and faster onset of avanafil would make it a good addition to the currently available phosphodiesterase type 5 inhibitors.

Two further trials funded by the manufacturers of avanafil are as yet not fully published. The trials have a similar design to that of the Goldstein et al trial ${ }^{14}$ except that the first one, REVIVE-Diabetes, ${ }^{16}$ focused on patients with diabetes and erectile dysfunction. This trial is also known as TA-302, and was first reported by the company marketing avanafil in 2010. The trial found that patients taking avanafil $100 \mathrm{mg}$ or $200 \mathrm{mg}$ as needed at least 30 minutes prior to sexual intercourse were able to complete intercourse at a higher rate than those on placebo. The second trial, TA-303, ${ }^{17}$ studied avanafil $100 \mathrm{mg}$ or $200 \mathrm{mg}$ in patients who had undergone a radical prostatectomy and were experiencing erectile dysfunction. Patients in the trial were found to be able to have successful intercourse more often with the active medication than with placebo. Side effects were similar to those in the other clinical studies.

\section{Discussion}

The three trials described above provide evidence of the effectiveness and safety of avanafil. Each of the trials was controlled, and used clinically relevant endpoints that would help a health care practitioner make decisions about which medication to use in patients with erectile dysfunction. The trials show that any of the marketed doses of avanafil are effective for a large proportion of patients with erectile dysfunction. The safety profile of the medication appears to be comparable with that of the other phosphodiesterase type 5 inhibitors, with similar side effects being present. The onset of action of avanafil appears to be shorter, at 15 minutes, which may help it find a niche in patients who are unable to plan sexual activity more than 15-30 minutes ahead of time. In vitro data suggest that avanafil is more selective for phosphodiesterase type 5 than the other phosphodiesterases, including phosphodiesterase type 11, which may reduce the vision issues that have been seen for other phosphodiesterase type 5 inhibitors with lower levels of specificity for phosphodiesterase 5 .

The trials do have weaknesses that inhibit interpretation of their data. The pharmacokinetic trial by Jung et al ${ }^{13}$ was conducted only in South Korea with Asian males as subjects. It is unclear if the data collected from this very uniform population are able to be extrapolated to larger non-Asian populations. Of concern is that this medication is being positioned in the market as having a rapid onset, as determined in this trial. Given the ability of other phosphodiesterase type 5 inhibitors to be used daily, the benefit of rapid action is not strictly necessary. Each of the trials testing efficacy compared avanafil with placebo. Although this is a "necessary evil" for preapproval trials, it will be difficult to determine if avanafil has a higher success rate in terms of improving the symptoms of erectile dysfunction over the currently available options. Finally, although this medication does have a higher selectivity for phosphodiesterase type 5 in vitro, it is concerning that a case of visual changes occurred during the trial in a treatment group. This has been a concern with other agents in this drug class, and it would be hoped that a "second-generation" phosphodiesterase type 5 inhibitor would not have this problem.

\section{Conclusion}

Avanafil is a new medication in the phosphodiesterase type 5 inhibitor class. The manufacturers plan to market avanafil as the first agent in the second generation of this class with a more rapid onset and a better side effect profile. It is apparent that avanafil is effective for the treatment of erectile dysfunction and from the 1300 patients who have taken the medication during clinical trials, it appears to be as safe as the other available agents in the class. However, it is unclear what role this agent will have in the four billion dollar per year marketplace for medications to treat erectile dysfunction. Previously, all of the agents in this class were similarly priced, but with sildenafil potentially becoming generically available later this year in some markets, patients 
may decide that the slight benefits of avanafil are not worth its extra cost.

One limitation to this review is the small amount of data and number of trials available. The other agents in this class have been on the world market for at least 10 years and have a proven track record of safety and efficacy, with some unpredicted side effects occurring. A new agent without such a long history may have difficulty finding a market share.

\section{Disclosure}

The authors report no conflicts of interest in this work.

\section{References}

1. US Food and Drug Administration. FDA approves Stendra for erectile dysfunction. Available from: http://www.fda.gov/NewsEvents/Newsroom/ PressAnnouncements/ucm302140.htm. Accessed May 1, 2012.

2. DeRogatis LR, Burnett AL. The epidemiology of sexual dysfunctions. J Sex Med. 2008;5:289-300.

3. Lue TF. Erectile dysfunction. N Engl J Med. 2000;342:1802-1813.

4. Batty G, Li Q, Czernichow S, et al. Erectile dysfunction and later cardiovascular disease in men with type 2 diabetes. $\mathrm{J} \mathrm{Am} \mathrm{Coll} \mathrm{Cardiol}$. 2010;56:1908-1913.

5. Dong JY, Zhang YH, Qin LQ. Erectile dysfunction and risk of cardiovascular disease: meta-analysis of prospective cohort studies. J Am Coll Cardiol. 2011;58:1378-1385.

6. Montorsi F, Adaikan G, Becher E, et al. Summary of the recommendations on sexual dysfunction in men. J Sex Med. 2010;7:3572-3588.
7. Gupta M, Kovar A, Meibohm B. The clinical pharmacokinetics of phosphodiesterase-5 inhibitors for erectile dysfunction. $J$ Clin Pharmacol. 2005;45:987-1003.

8. Gingell C, Sultana S, Wulff M, Gepi-Attee S. Duration of action of sildenafil citrate in men with erectile dysfunction. $J$ Sex Med. 2004;1: 179-184.

9. Limin M, Johnsen N, Hellstrom WJ. Avanafil, a new rapid-onset phosphodiesterase 5 inhibitor for the treatment of erectile dysfunction. Expert Opin Investig Drugs. 2010;19:1427-1437.

10. Rosen R, Kostis J. Overview of phosphodiesterase 5 inhibition in erectile dysfunction. Am J Cardiol. 2003;92(9A):9M-18M.

11. Alwaal A, Al-Mannie R, Carrier S. Future prospects in the treatment of erectile dysfunction: focus on avanafil. Drug Des Devel Ther. 2011; 5:435-443.

12. Evans JD, Pace K, Evans EW. Natural therapies used by adult men for the treatment of erectile dysfunction, benign prostatic hyperplasia, and for augmenting exercise performance. J Pharm Pract. 2011;2:323-331.

13. Jung J, Choi S, Cho S, et al. Tolerability and pharmacokinetics of avanafil, a phosphodiesterase type 5 inhibitor: a single- and multiple-dose, doubleblind, randomized, placebo-controlled, dose-escalation study in healthy Korean male volunteers. Clin Ther. 2010;32:1178-1187.

14. Goldstein I, McCullough A, Jones L, et al. A randomized, double-blind, placebo-controlled evaluation of the safety and efficacy of avanafil in subjects with erectile dysfunction. J Sex Med. 2012;9:1122-1133.

15. Zhao C, Kim S, Yan D, et al. Efficacy and safety of avanafil for treating erectile dysfunction: results of a multicentre, randomized, double-blind, placebo-controlled trial. BJU Int. March 27, 2012. [Epub ahead of print.]

16. Release about TA-302, http://ir.vivus.com/releasedetail.cfm?ReleaseID= 476930. Accessed May 1, 2012.

17. Release aboutTA-303, http://ir.vivus.com/releasedetail.cfm?ReleaseID= 585757. Accessed May 1, 2012.
Vascular Health and Risk Management

\section{Publish your work in this journal}

Vascular Health and Risk Management is an international, peerreviewed journal of therapeutics and risk management, focusing on concise rapid reporting of clinical studies on the processes involved in the maintenance of vascular health; the monitoring, prevention and treatment of vascular disease and its sequelae; and the involvement of

\section{Dovepress}

metabolic disorders, particularly diabetes. This journal is indexed on PubMed Central and MedLine. The manuscript management system is completely online and includes a very quick and fair peer-review system, which is all easy to use. Visit http://www.dovepress.com/ testimonials.php to read real quotes from published authors. 